\title{
EL CARIOTIPO DE BOMAREA PATINII BAKER SUBSP. PATINII (ALSTROEMERIACEAE)
}

\section{THE KARYOTYPE OF BOMAREAPATINII BAKER SUBSP. PATINII (ALSTROEMERIACEAE)}

\author{
Carlos M. Baeza ${ }^{1}$, Fernando Alzate ${ }^{2}$, María Negritto ${ }^{1}$ \& Eduardo Ruiz ${ }^{1}$ \\ ${ }^{1}$ Departamento de Botánica, Universidad de Concepción, Concepción, Chile; ${ }^{2}$ Posgrado de Biología, Universidad de \\ Antioquia, Apartado Aéreo 1226, Medellín, Antioquia, Colombia. \\ cbaeza@udec.cl
}

\begin{abstract}
The karyotype of Bomarea patinii Baker subsp. patinii from Colombia was examined. The species has a karyotype $2 \mathrm{n}$ $=2 \mathrm{x}=18$, with a haploid formula $2 \mathrm{~m}+1 \mathrm{sm}+5 \mathrm{st}+1 \mathrm{t}$-sat. The reported karyotype is asymmetric $($ AsI $\%=75.51)$. The most of the chromosomes are acrocentric. This results are compared with other species of Bomarea from South America.
\end{abstract}

Bomarea Mirb. (Alstroemeriaceae) comprende alrededor de 100 especies (Sanso \& Hunziker 1998), con una distribución neotropical y austroamericana (Hofreiter \& Rodríguez 2006), que se extiende desde México y Antillas hasta Argentina y Chile (PalmaRojas et al. 2007). B. patinii Baker subsp. patinii está ampliamente distribuida en los bosques andinos de Colombia, Venezuela, Ecuador y Perú, y crece en sectores abiertos, incluso a orillas de caminos (Alzate 2005). Sus flores son de color rojo intenso agrupadas en una umbela racemosa (Fig. 1).

Los estudios citogenéticos en Bomarea son escasos. Sin embargo, en los trabajos de Sanso \& Hunziker (1998) y Palma-Rojas et al. (2007) queda de manifiesto la importancia de los estudios cromosómicos en Bomarea a nivel taxonómico y evolutivo en relación a los otros géneros de la familia (Leontochir, Alstroemeria). En esta comunicación se documenta el cariotipo de $B$. patinii subsp. patinii y se compara con otras especies del género de distribución más austral.

Se estudió una población de Bomarea patinii subsp. patinii proveniente de Colombia, Departamento de Cundinamarca, Municipio de Sibaté, Alto de San Miguel, 2.650 m (4'24'N/74'22'W), 12-XI-2005, F. Alzate 2894, depositados en el Herbario HUA de la Universidad de Antioquia, en Medellín, Colombia. Las plantas fueron cultivadas en invernadero a partir de semillas.
El estudio de los cromosomas y mediciones se realizaron siguiendo la metodología propuesta por Baeza et al. (2000, 2001, 2004, 2007), la cual consistió en puntas de raíces de 1-2 cm de longitud, obtenidas a partir de plantas cultivadas en invernadero fueron pretratadas con una solución de 8-hidroxiquinoleina $(2 \mathrm{mM})$ por $24 \mathrm{~h} \mathrm{a} 4{ }^{\circ} \mathrm{C}$. Luego, se fijaron en etanol / ácido acético (3:1) por 24 h. Posteriormente, se hizo una hidrólisis ácida con $\mathrm{HCl}$ 0,5 N durante 20 min a $42{ }^{\circ} \mathrm{C}$. Luego se lavó el material con agua destilada dos veces para eliminar el exceso de HCl. Finalmente, se hizo el aplastado de la punta de la raíz en una gota de orceína acética al 1\%. Para la población analizada (10 placas metafásicas) se determinó el índice de asimetría del cariotipo (AsI \%) definido por Arano y Saito (1980). Los cromosomas se clasificaron de acuerdo a Levan et al. (1964).

Bomarea patinii subsp. patinii presenta $2 \mathrm{n}=2 \mathrm{x}$ $=18$ cromosomas, con un cariotipo asimétrico y un complemento cromosómico con 2 pares metacéntricos (pares 2 y 5), 1 par submetacéntrico (par 4), 5 pares subtelocéntricos (pares 3, 6-9) y 1 par telocéntrico con satélite (par 1) (Fig. 2, Tabla I). El cociente entre el par más largo y el par más corto (R) fue 2,07 y el índice de asimetría del cariotipo de Arano y Saito (AsI \%) fue de 75,51, lo que está indicando un alto grado de asimetría del cariotipo, con cromosomas en su mayoría acrocéntricos. Sanso \& Hunziker (1998) describen el cariotipo de B. boliviensis 
Baker y B. edulis (Tussac) Herb. provenientes de Argentina, y Palma-Rojas et al. (2007) describen el cariotipo de $B$. involucrosa (Herb.) Baker y $B$. salsilla (L.) Mirb. de Chile. En ambos trabajos se indica un $2 \mathrm{n}=18$ cromosomas para cada especie, sin presencia de poliploides y con el cromosoma 5 siempre metacéntrico, lo que pareciera ser una condición permanente en este género que podría ser utilizado como un cromosoma marcador. Hunziker \& Xifreda (1990) encontraron tetraploidia en $B$. hirtella (Kunth) Herb. constituyendo este el único caso reportado para el género. El análisis del cariotipo en $B$. patinii subsp. patinii también corrobora que el cromosoma 5 (Fig. 2 B, C) es metacéntrico. Al comparar la fórmula cariotípica entre las 5 especies analizadas ninguna de ellas se repite y cada especie presenta una fórmula característica, aunque sólo se analizó una población por especie. Es probable que al estudiar un mayor número de poblaciones se podrían encontrar mayores similitudes entre ellas o al contrario confirmar la diferencia ya existente. Sólo $B$. boliviensis presenta gran similitud con $B$. patinii subsp. patinii. En esta subespecie el cromosoma 1 es telocéntrico y presenta un satélite en la porción terminal del brazo corto (Fig. 2 B). Esta condición se documenta por primera vez para el género Bomarea. Sin embargo, Palma-Rojas et al. (2007) señalan para Leontochir ovallei Phil., especie de Alstroemeriaceae endémica del norte de Chile, la presencia de un satélite en el brazo corto del cromosoma 2 (este cromosoma también podría tratarse del par 1), que también es telocéntrico. Esta situación estaría apoyando la inclusión de Leontochir en Bomarea, tema ya planteado por Ravenna (2000) y Hofreiter (2005). La longitud total diploide de los cromosomas (LTC) fue de $123,4 \mu$ m para B. patinii subsp. patinii, valor similar a las otras especies de Bomarea estudiadas. B. salsilla, especie endémica de Chile, presenta un valor muy bajo de LTC comparado con las otras especies de Bomarea, lo que está indicando que sus cromosomas son de pequeño tamaño. Sería interesante y necesario analizar el cariotipo en otras poblaciones de esta especie en Chile, para definir si esta condición se mantiene o sólo depende de la distribución geográfica de ella.

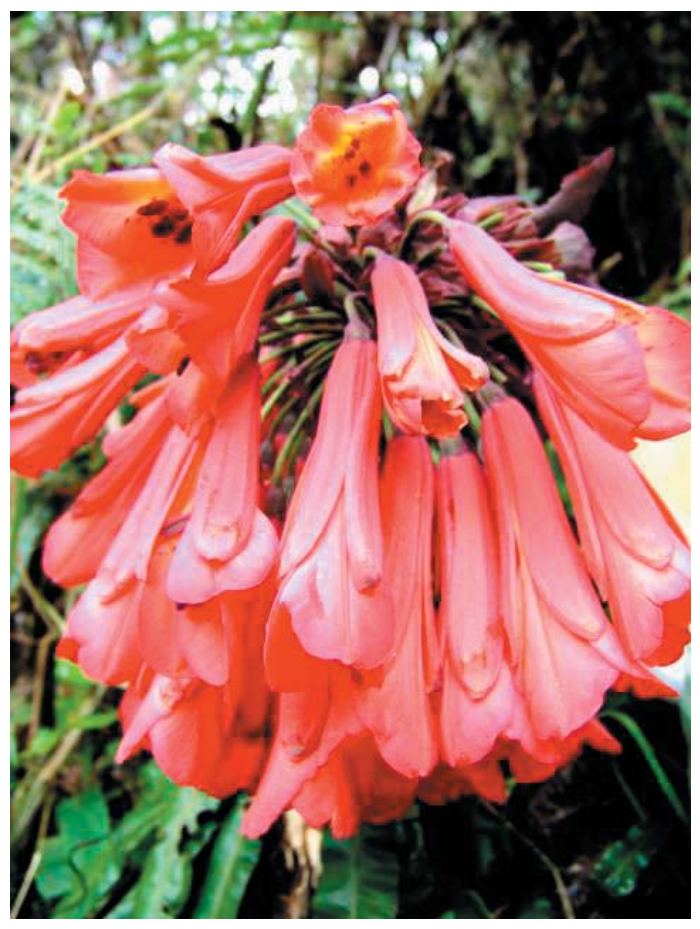

FIGURA 1. Fotografía de las flores de Bomarea patinii subsp. patinii (Alzate 2894).

FIgURE 1. Photography of the flower of Bomarea patinii subsp. patinii (Alzate 2894). 


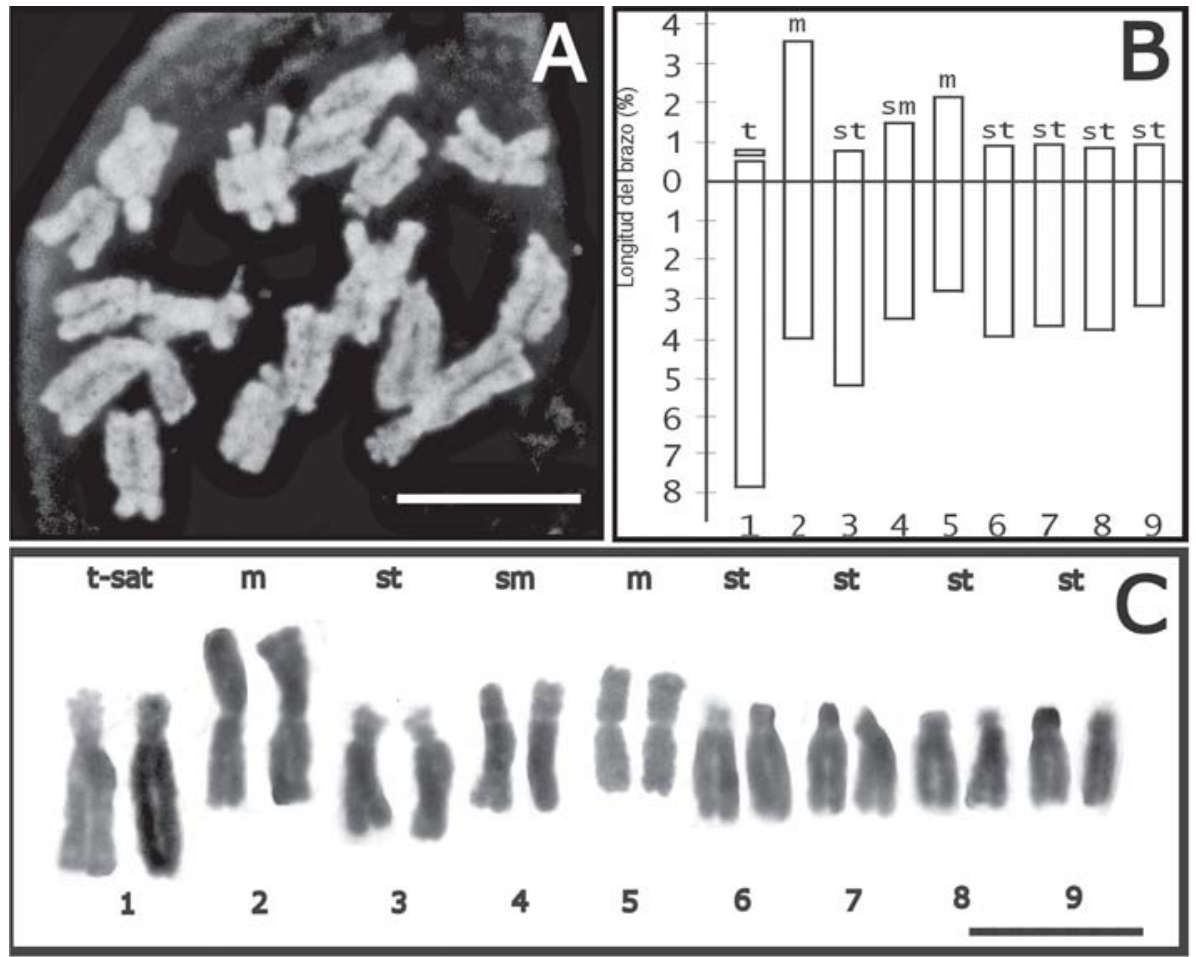

Figura 2. A. Cromosomas en metafase de Bomarea patinii subsp. patinii $(2 \mathrm{n}=18)$. B. Idiograma del complemento haploide (los cromosomas se han ordenado de acuerdo a su tamaño decreciente). C. Cariotipo obtenido del análisis de 10 placas metafásicas. Las escalas corresponden a $10 \mu \mathrm{m}$.

Figure 2. A. Metaphasic chromosomes of Bomarea patinii subsp. patinii $(2 \mathrm{n}=18)$. B. Ideogram of the haploid chromosomes complement (the chromosomes have been ordered according to decreasing size). C. Karyotype using 10 metaphasic plates. Scales $=10 \mu \mathrm{m}$.

Tabla I. Mediciones cromosómicas e índice braquial (r) de Bomarea patinii subsp. patinii. Se detallan las longitudes promedio como porcentaje de la longitud del genoma haploide de 10 metafases.

TABLE I. Chromosomes measurements and braquial index (r) of Bomarea patinii subsp. patinii. Average length of chromosomes calculated in percent of the mean haploid genome length of 10 metaphases.

\begin{tabular}{|c|c|c|c|c|c|c|}
\hline $\begin{array}{c}\text { Par } \\
\text { cromosómico }\end{array}$ & $\begin{array}{l}\text { Long. brazo largo } \\
\text { (\%) } \pm \text { S.D. }\end{array}$ & $\begin{array}{l}\text { Long. brazo corto } \\
\text { (\%) } \pm \text { S.D. }\end{array}$ & $\begin{array}{l}\text { Long. total } \\
\text { relativa (\%) }\end{array}$ & $\begin{array}{l}\text { Long. total absoluta } \\
\qquad(\mu \mathrm{m})\end{array}$ & $r$ & $\begin{array}{c}\text { Tipo } \\
\text { de cromosoma }\end{array}$ \\
\hline 1 & $7,77 \pm 0,20$ & $0,73 \pm 0,14$ & 8,50 & 10,50 & 10,6 & t-sat \\
\hline 2 & $3,99 \pm 0,18$ & $3,57 \pm 0,14$ & 7,56 & 9,30 & 1,1 & $\mathrm{~m}$ \\
\hline 3 & $5,19 \pm 0,20$ & $0,78 \pm 0,10$ & 5,97 & 7,37 & 6,7 & st \\
\hline 4 & $3,49 \pm 0,20$ & $1,49 \pm 0,10$ & 4,98 & 6,15 & 2,3 & $\mathrm{sm}$ \\
\hline 5 & $2,78 \pm 0,10$ & $2,15 \pm 0,14$ & 4,93 & 6,09 & 1,3 & $\mathrm{~m}$ \\
\hline 6 & $3,94 \pm 0,10$ & $0,91 \pm 0,10$ & 4,85 & 5,95 & 4,3 & st \\
\hline 7 & $3,69 \pm 0,10$ & $0,94 \pm 0,18$ & 4,63 & 5,72 & 3,9 & st \\
\hline 8 & $3,77 \pm 0,25$ & $0,75 \pm 0,20$ & 4,52 & 5,58 & 5,0 & st \\
\hline 9 & $3,16 \pm 0,20$ & $0,94 \pm 0,15$ & 4,10 & 5,06 & 3,4 & st \\
\hline
\end{tabular}


Gayana Bot. 65(2), 2008

\section{AGRADECIMIENTOS}

Se agradece al proyecto Fondecyt 1070520 y al Departamento de Botánica de la Universidad de Concepción por las facilidades otorgadas.

\section{BIBLIOGRAFIA}

Alzate, F. 2005. Bomarea patinii ssp. umbellata (Alstroemeriaceae), a new subspecies of Bomarea. Harvard Papers in Botany 10(1): 53-56.

Arano, H. \& H. SaIto. 1980. Cytological studies in family Umbelliferae 5 . Karyotypes of seven species in subtribe Seselinae. La Kromosomo 2: 471-480.

Baeza, C., J. Grau, M. Vosyka, T. Stuessy \& H. Weiss. 2000. Recuentos cromosómicos en especies de Hypochaeris L. de Chile. Gayana Botánica 57(1): 105-106.

Baeza, C., G. Kottirsch, J. Espejo \& R. Reinoso. 2001. Recuentos cromosómicos en plantas chilenas. I. Gayana Botánica 58(2): 133-137.

Baeza, C., M. Vosyka \& T. Stuessy. 2004. Recuentos cromosómicos en plantas que crecen en Chile. II. Darwiniana 42(1-2): 25-29.
Baeza, C., E. Ruiz \& M. Negritto. 2007. El número cromosómico de Phycella australis Ravenna (Amaryllidaceae). Gayana Botánica 64(1): 117-120.

Hofreiter, A. 2005. The genus Bomarea (Alstroemeriaceae) in Bolivia and southern South America. Harvard Papers in Botany 9: 343-374.

Hofreiter, A. \& E. Rodríguez. 2006. The Alstroemeriaceae in Peru and neighbouring areas. Revista Peruana de Biología 13: 5-69.

HunziKER, J. \& C. XifRedA. 1990. Chromosomes studies in Bomarea and Alstroemeria (Alstroemeriaceae). Darwiniana 30: 179-183.

LeVAn, A., K. Fredga \& A. SANDBERg. 1964. Nomenclature for centromeric position on chromosomes. Hereditas 52: 201-220.

Palma-Rojas, C., P. Jara-Seguel \& E. Von Brand. 2007. Karyological studies in Chilean species of Bomarea and Leontochir (Alstroemeriaceae). New Zealand Journal of Botany 45: 299-303.

Ravenna, P. 2000. New or interesting AlstroemeriaceaeIII. Onira 5: 35-41.

SAnso, A. \& J. HunziKer. 1998. Karyological studies in Alstroemeria and Bomarea (Alstroemeriaceae). Hereditas 129: 67-74.

Recibo:11.03.08

Aceptado: 15.05 .08 